\title{
Voltages and Currents in Output Circuits of Three-Phase Diode Rectifiers
}

\author{
Piotr Olszowiec* \\ Erea Ltd.
}

\begin{abstract}
Operating parameters such as voltages, leakage, and ground fault currents are decisive for a correct and safe operation of low-voltage unearthed networks with diode rectifiers. The knowledge of the maximum possible values of these parameters is necessary for correct assessment of numerous hazards. So far, no simple methods for determining the highest levels of these parameters in AC/DC IT three-phase networks have been available. The paper presents a new approach to the evaluation of the variation ranges of diode rectifiers' voltages and currents with the help of Mathcad.
\end{abstract}

Keywords - Diode rectifiers, ground fault current, insulation resistance, leakage current, output voltage, three-phase networks.

\section{INTRODUCTION}

Three-phase low voltage networks with unearthed neutral (see Fig. 1) are commonly applied for the supply of rectifiers feeding power and/or auxiliary circuits. An important task of these systems (AC/DC IT) operation is maintaining their insulation-to-ground at required level. Network-to-ground insulation resistance exerts influence on such operating parameters as phase voltages, leakage and ground fault currents [1], which are decisive for a safe and correct operation of these systems. An excessive phase voltage rise (overvoltage) increases the risk of insulation breakdown, whereas high leakage and ground fault currents may cause fire or explosion. Also the risks of the electric shock of humans as well as of incorrect relays operation must be taken into account [2].

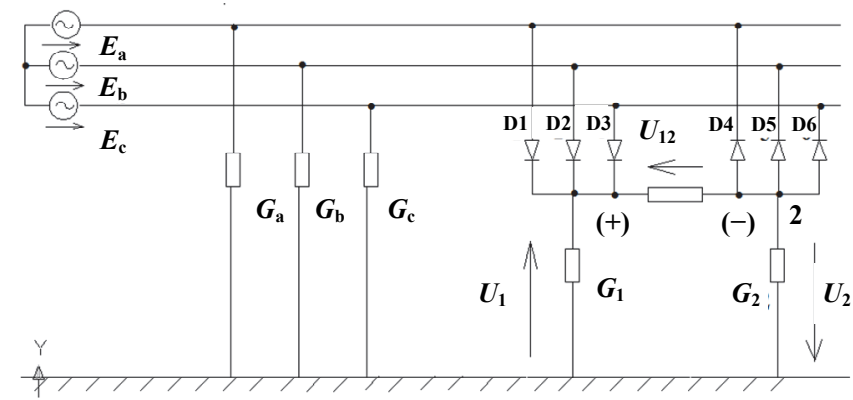

Fig. 1. Equivalent circuit of a three-phase IT network with a diode rectifier (with the ground capacitances at $\mathrm{AC}$ and DC sides neglected).
The knowledge of the maximum possible values of network operating parameters is necessary for the assessment of the mentioned hazards. Some analysis of these parameters variation ranges at AC side of these "mixed" systems has been presented in [3]. However, complete theory on voltages and currents in diode rectifiers' output circuits is still commonly unavailable and no research results of the highest levels of operating parameters have been published so far.

\section{Diode Rectifier OUtPut Voltage}

Three-phase diode bridge rectifier output voltage consists predominantly of a DC component. The mean value of the rectified voltage is given by the well-known formula [1]

$$
U_{12 \text { mean }}=\frac{3 \sqrt{2} E_{\mathrm{ph} \text {-ph }}}{\pi}
$$

valid for symmetrical phase-to-phase voltages $E_{\mathrm{ph} \text {-ph }}$ (RMS values) of the supplying source.

For asymmetrical supply, the output voltage mean value can be calculated from a general expression [4]:

$$
U_{12 \text { mean }}=\frac{\sqrt{2}\left(E_{\mathrm{ab}}+E_{\mathrm{bc}}+E_{\mathrm{ca}}\right)}{\pi},
$$

where $E_{\mathrm{ab}}, E_{\mathrm{bc}}, E_{\mathrm{ca}}$ are RMS values of network phase-to-phase voltages.

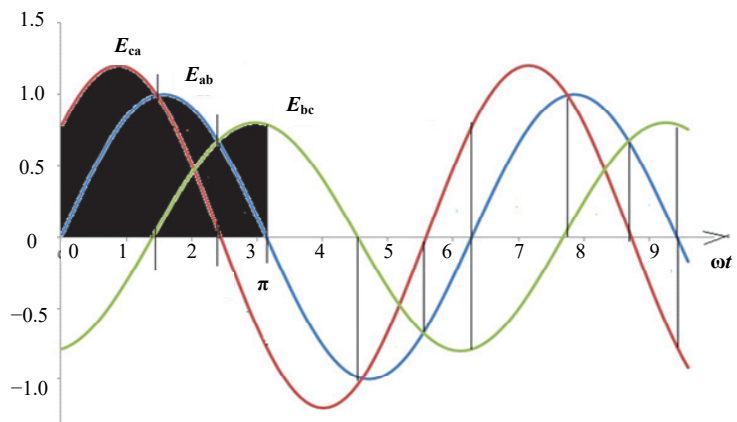

Fig. 2. Waveforms of phase-to-phase asymmetrical voltages feeding a diode rectifier (black area represents the output voltage for half of period).

This formula can be derived in the following way:

\footnotetext{
* Corresponding author.

E-mail address: olpio@o2.pl
} 
$U_{12 \text { mean }}=\frac{\sqrt{2}}{\pi}\left(\int_{0}^{\beta} E_{c a} \sin (x+\gamma) \mathrm{d} x+\int_{\beta}^{\pi-y}\left[E_{\mathrm{bc}} \sin (x-\beta)+E_{\mathrm{ca}} \sin (x+\gamma)\right] \mathrm{d} x+\int_{\pi-\gamma}^{\pi} E_{\mathrm{bc}} \sin (x-\beta) \mathrm{d} x\right)=$

$=\frac{\sqrt{2} E_{\mathrm{ca}}}{\pi} \int_{0}^{\pi-y} \sin (x+\gamma) \mathrm{d} x+\frac{\sqrt{2} E_{\mathrm{bc}}}{\pi} \int_{\beta}^{\pi} \sin (x-\beta) \mathrm{d} x=\frac{\sqrt{2} E_{\mathrm{ca}}}{\pi}[\cos \gamma+1]+\frac{\sqrt{2} E_{\mathrm{bc}}}{\pi}[1+\cos \beta]=$

$=\frac{\sqrt{2}\left(E_{\mathrm{ab}}+E_{\mathrm{bc}}+E_{\mathrm{ca}}\right)}{\pi}$

$$
\text { as } E_{\mathrm{ab}} \sin \left(\frac{\pi}{2}\right)=E_{\mathrm{bc}} \sin \left(\frac{\pi}{2}-\beta\right)+E_{\mathrm{ca}} \sin \left(\frac{\pi}{2}+\gamma\right) \text {. }
$$

It would be interesting to determine the accurate output voltage RMS value $U_{12 \mathrm{RMS}}$ as well as its AC component $U_{12 \mathrm{AC}}$. For a symmetrical three-phase supply, these parameters are equal to

$$
\begin{aligned}
& U_{12 \mathrm{RMS}}=E_{\mathrm{ph}-\mathrm{ph}} \sqrt{\frac{3 \sqrt{3}+2 \pi}{2 \pi}}, \\
& U_{12 \mathrm{AC}}=\sqrt{U_{12 \mathrm{RMS}}^{2}-U_{12 \text { mean }}^{2}},
\end{aligned}
$$

According to formulas (5) and (6), the harmonics content $U_{12 \mathrm{AC}} / U_{12 \mathrm{RMS}}$ in output voltage is $4.2 \%$.

\section{RECTIFIER POLES-TO-GROUND VOLTAGES VARIATION RANGES}

For the analysis of the variation of pole (to ground) voltages RMS values, it is convenient to make the following assumption. Usually, network-to-ground capacitances can be neglected, which clearly simplifies further analysis. Let the time functions of symmetrical source phase voltages be as follows:

$$
\begin{gathered}
e_{\mathrm{a}}(t)=E_{\mathrm{m}} \cdot \sin \omega t, e_{\mathrm{b}}(t)=E_{\mathrm{m}} \cdot \sin \left[\omega\left(t-\frac{T}{3}\right)\right], \\
e_{\mathrm{c}}(t)=E_{\mathrm{m}} \cdot \sin \left[\omega\left(t-\frac{2 T}{3}\right)\right] .
\end{gathered}
$$

The RMS value of a selected pole-to-ground voltage, e. g., negative pole 2 , can be calculated with the help of expressions valid for the respective intervals of diode commutation. For convenience, the equivalent insulation conductances are designated at AC side as $G_{\mathrm{iAC}}=G_{\mathrm{a}}+G_{\mathrm{b}}+G_{\mathrm{c}}$ and at DC side as $G_{\mathrm{iDC}}=G_{1}+G_{2}$. In consecutive intervals of the rectifier diodes commutation, the momentary values of negative pole voltage $u_{2}(t)$ are given by the following formulas:

\section{$T / 12-3 T / 12$ (D1, D5 diodes conduct):}

$u_{2}\left(t_{1,3}=E \frac{\left(G_{\mathrm{a}}+G_{1}\right) \sin (\omega t)+\left(G_{\mathrm{b}}+G_{2}\right) \sin \left(\omega t-\frac{2 \pi}{3}\right)+G_{\mathrm{c}} \cdot \sin \left(\omega t-\frac{4 \pi}{3}\right)}{G_{\mathrm{iAC}}+G_{\mathrm{iDC}}}-E \sin \left(\omega t-\frac{2 \pi}{3}\right) ;\right.$

$3 T / 12-5 T / 12$ (D1, D6 diodes conduct):

$$
u_{2}(t)_{3,5}=E \frac{\left(G_{\mathrm{a}}+G_{1}\right) \sin (\omega t)+G_{\mathrm{b}} \sin \left(\omega t-\frac{2 \pi}{3}\right)+\left(G_{\mathrm{c}}+G_{2}\right) \sin \left(\omega t-\frac{4 \pi}{3}\right)}{G_{\mathrm{iAC}}+G_{\mathrm{iDC}}}-E \sin \left(\omega t-\frac{4 \pi}{3}\right) ;
$$

$5 T / 12-7 T / 12$ (D2, D6 diodes conduct):

$u_{2}(t)_{5,7}=E \frac{G_{\mathrm{a}} \sin (\omega t)+\left(G_{\mathrm{b}}+G_{1}\right) \sin \left(\omega t-\frac{2 \pi}{3}\right)+\left(G_{\mathrm{c}}+G_{2}\right) \sin \left(\omega t-\frac{4 \pi}{3}\right)}{G_{\mathrm{iAC}}+G_{\mathrm{iDC}}}-E \sin \left(\omega t-\frac{4 \pi}{3}\right)$

7T/12-9T/12 (D2, D4 diodes conduct):

$u_{2}(t)_{7,9}=E \frac{\left(G_{\mathrm{a}}+G_{2}\right) \sin (\omega t)+\left(G_{\mathrm{b}}+G_{1}\right) \sin \left(\omega t-\frac{2 \pi}{3}\right)+G_{\mathrm{c}} \sin \left(\omega t-\frac{4 \pi}{3}\right)}{G_{\mathrm{iAC}}+G_{\mathrm{iDC}}}-E \sin (\omega t)$

9T/12 - 11T/12 (D3, D4 diodes conduct):

$u_{2}\left(t_{9,11}=E \frac{\left(G_{\mathrm{a}}+G_{2}\right) \sin (\omega t)+G_{\mathrm{b}} \sin \left(\omega t-\frac{2 \pi}{3}\right)+\left(G_{\mathrm{c}}+G_{1}\right) \sin \left(\omega t-\frac{4 \pi}{3}\right)}{G_{\mathrm{iAC}}+G_{\mathrm{iDC}}}-E \sin (\omega t) ;\right.$

$11 T / 12-13 T / 12$ (D3, D5 diodes conduct):

$u_{2}(t)_{1,1,13}=E \frac{G_{\mathrm{a}} \sin (\omega t)+\left(G_{\mathrm{b}}+G_{2}\right) \sin \left(\omega t-\frac{2 \pi}{3}\right)+\left(G_{\mathrm{c}}+G_{1}\right) \sin \left(\omega t-\frac{4 \pi}{3}\right)}{G_{\mathrm{iAC}}+G_{\mathrm{iDC}}}-E \sin \left(\omega t-\frac{2 \pi}{3}\right)$.

According to definition, the RMS value of $U_{2}$ voltage is

$$
\frac{U_{2 \mathrm{rms}}=\sqrt{\left[\frac{1}{T}\left(\int_{T / 12}^{3 T / 12} u_{2(1,3)}^{2} \mathrm{~d} t+\int_{3 T / 12}^{5 T / 12} u_{2(3,5)}^{2} \mathrm{~d} t+\int_{5 T / 12}^{7 T / 12} u_{2(5,7)}^{2} \mathrm{~d} t\right)\right.}}{\left.+\frac{1}{T}\left(\int_{7 T / 12}^{9 T / 12} u_{2(7,9)}^{2} \mathrm{~d} t+\int_{9 T / 12}^{11 T / 12} u_{2(9,11)}^{2} \mathrm{~d} t+\int_{11 T / 12}^{13 T / 12} u_{2(11,13)}^{2} \mathrm{~d} t\right)\right]} .
$$

It can be shown that the RMS value of pole 2 voltage assumes its maximum when the entire $\mathrm{AC}$ side insulation leakage conductance is lumped at one phase only, i. e., $G_{\mathrm{a}}=G_{\mathrm{iAC}}$, $G_{\mathrm{b}}=G_{\mathrm{iAC}}$, or $G_{\mathrm{c}}=G_{\mathrm{iAC}}$, whereas the entire $\mathrm{DC}$ side insulation leakage conductance is lumped at the rectifier's positive pole, i. e., $G_{1}=G_{\text {iDC. }}$. The function attains its minimum when $G_{\mathrm{a}}=G_{\mathrm{b}}=G_{\mathrm{c}}=G_{\mathrm{iAC}} / 3$ and $G_{2}=G_{\mathrm{iDC}}$. Both maximum and minimum values of $U_{2 \mathrm{rms}}$ depend on the ratio $G_{\mathrm{iAC}} / G_{\mathrm{iDC}}$. The dependence of this voltage referred to the mean value of the rectifier output voltage $U_{2 \mathrm{rms}} / U_{12 \text { mean }}$ on $G_{\mathrm{iAC}} / G_{\mathrm{iDC}}=$ $R_{\mathrm{iDC}} / R_{\mathrm{iAC}}$ is plotted in Fig. 3. The highest value 1 is obtained for $R_{\mathrm{iDC}} / R_{\mathrm{iAC}}=0$.

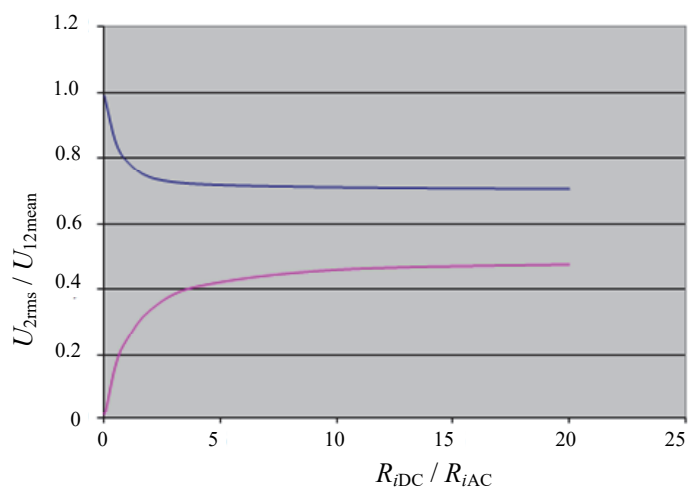

Fig. 3. Dependence of the maximum and minimum pole-to-ground voltage RMS value referred to the rectifier mean output voltage on the $R_{\mathrm{iDC}} / R_{\mathrm{iAC}}$ ratio. 
Rectifier poles-to-ground voltages contain mainly the DC component. These mean values can be expressed by the following formulas (derived in [5]):

$$
\begin{aligned}
& U_{1 \text { mean }}=\frac{G_{\mathrm{a}}+G_{\mathrm{b}}+G_{\mathrm{c}}+2 G_{2}}{G_{\mathrm{a}}+G_{\mathrm{b}}+G_{\mathrm{c}}+G_{1}+G_{2}} \cdot \frac{U_{12 \text { mean }}}{2}, \\
& U_{2 \text { mean }}=\frac{G_{\mathrm{a}}+G_{\mathrm{b}}+G_{\mathrm{c}}+2 G_{1}}{G_{\mathrm{a}}+G_{\mathrm{b}}+G_{\mathrm{c}}+G_{1}+G_{2}} \cdot \frac{U_{12 \text { mean }}}{2} .
\end{aligned}
$$

As in the case of RMS values, the mean voltage of pole 2 assumes its maximum when the entire $\mathrm{AC}$ side insulation leakage conductance is lumped at one phase only, i. e., $G_{\mathrm{a}}=G_{\underline{\mathrm{iAC}}}, G_{\mathrm{b}}=G_{\mathrm{iAC}}$, or $G_{\mathrm{c}}=G_{\mathrm{iAC}}$, whereas the entire DC side insulation leakage conductance is lumped at the rectifier's positive pole, i. e., $G_{1}=G_{\text {iDC. }}$. The function attains its minimum when $G_{\mathrm{a}}=G_{\mathrm{b}}=G_{\mathrm{c}}=G_{\mathrm{iAC}} / 3$ and $G_{2}=G_{\mathrm{iDC}}$. Both maximum and minimum values of $U_{2 \text { mean }}$ depend on the ratio $R_{\mathrm{iDC}} / R_{\mathrm{iAC}}$ (Fig. 4).

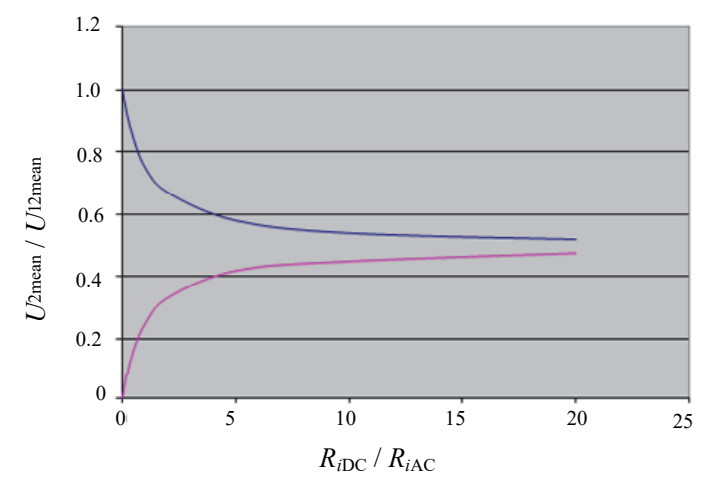

Fig. 4. Dependence of the maximum and minimum pole-to-ground voltage mean value (referred to the rectifier mean output voltage) on the $R_{\mathrm{iDC}} / R_{\mathrm{iAC}}$ ratio.

Comparing Figs 3 and 4, it can be concluded that the RMS value of pole voltage is approximately a dozen percent higher than its mean value, which is caused by the harmonics content.

\section{LEAKAGE AND GROUND FAULT CURRENTS}

High leakage current, i. e., active component of the current flowing through pole-to-ground insulation conductances, poses the threat of excessive temperature rise in places with lowered insulation level. This may cause fire or explosion in the presence of flammable or explosive substances. High leakage and ground fault currents mean also an increased electric shock hazard for people. Another risk is an incorrect operation of DC relays fed from rectifiers.

Leakage current (RMS value) from, for example, negative pole, is given by the following formula:

$$
I_{2 \text { RMSleak }}=U_{2 \mathrm{RMS}} G_{2} \text {. }
$$

As previously, it can be shown that RMS value of pole 2 leakage current assumes its maximum when the entire AC side insulation leakage conductance is lumped at one phase only, i. e., $G_{\mathrm{a}}=G_{\mathrm{iAC}}$, and the entire DC side insulation leakage conductance $G_{\mathrm{iDC}}$ is lumped at one of rectifier poles. The results of maximum leakage currents measurements for a rectifier with output mean voltage $U_{12 \text { mean }}=1 \mathrm{~V}$ are given in Table I.

TABLE I

Maximum Leakage CurRents [ $\mu \mathrm{A}$ ] AT ANy Pole For $\mathrm{U}_{12 \text { Mean }}=1 \mathrm{~V}$

\begin{tabular}{|r|r|r|r|r|r|}
\hline $\begin{array}{r}R_{\mathrm{iDC}}, \\
\mathrm{k} \Omega\end{array}$ & 10 & 30 & 50 & 70 & 90 \\
$R_{\mathrm{iAC}}, \mathrm{k} \Omega$ & & & & & \\
\hline 10 & 33.0 & 17.0 & 10.5 & 8.0 & 6.5 \\
\hline 30 & 28.0 & 12.5 & 9.0 & 6.5 & 5.5 \\
\hline 50 & 27.0 & 11.5 & 8.0 & 5.6 & 5.0 \\
\hline 70 & 26.5 & 10.7 & 7.0 & 5.0 & 4.3 \\
\hline 90 & 26.0 & 10.0 & 6.0 & 4.5 & 4.0 \\
\hline
\end{tabular}

The surface plotted in Fig. 6 according to formulas (14), (16) presents the dependence of the maximum leakage current $I_{2 \text { RMSleak }}$ on insulation equivalent resistances. This diagram (and Table I with the help of linear interpolation) can be used for an approximate, quick evaluation of the sought maximum level of leakage current at DC side.

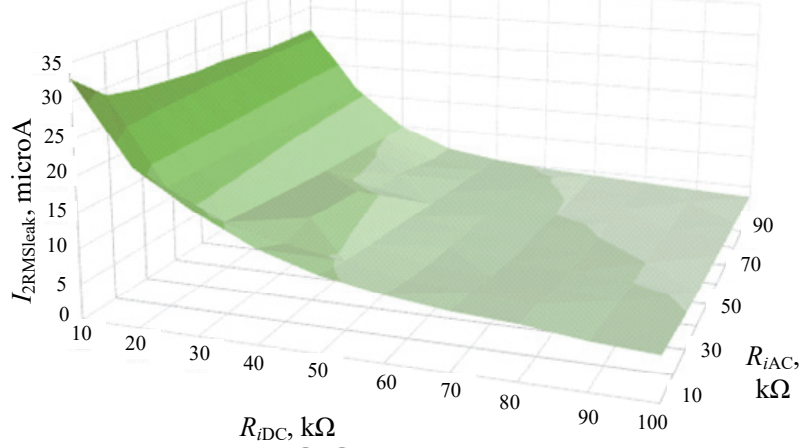

Fig. 5. Dependence of any pole maximum leakage current (RMS value) for the rectifier output voltage $U_{12 \text { mean }}=1 \mathrm{~V}$ on the $R_{\mathrm{iDC}}$ and $R_{\mathrm{iAC}}$ ratio.

In a similar way, the maximum ground fault current at DC side is evaluated. Also in this case, the pole-to-ground current assumes its maximum when entire AC side insulation leakage conductance is lumped at one phase only and the entire DC side insulation leakage conductance is lumped at one pole.

The results of maximum short circuit current measurements in a network with $U_{12 \text { mean }}=1 \mathrm{~V}$ are given in Table II. In Fig. 6, there is presented the dependence of DC side maximum "dead" ground fault current on $R_{\mathrm{iDC}}$ and $R_{\mathrm{iAC}}$ ratio.

TABLE II

MAXIMUM SHORT CIRCUIT CURRENT $[\mu \mathrm{A}]$ AT ANY POLE FOR $U_{12 \text { MEAN }}=1 \mathrm{~V}$

\begin{tabular}{|c|r|r|r|r|r|}
\hline $\begin{array}{c}R_{\mathrm{iDC}}, \\
\mathrm{k} \Omega\end{array}$ & 10 & 30 & 50 & 70 & 90 \\
\hline$R_{\mathrm{iAC}, \mathrm{k} \Omega}$ & 150 & 84 & 69 & 64 & 60 \\
\hline 30 & 116 & 53 & 37 & 32 & 28 \\
\hline 50 & 109 & 45 & 29 & 24 & 21 \\
\hline 70 & 106 & 43 & 26 & 20 & 17.5 \\
\hline 90 & 104 & 41 & 24.5 & 18 & 16 \\
\hline
\end{tabular}




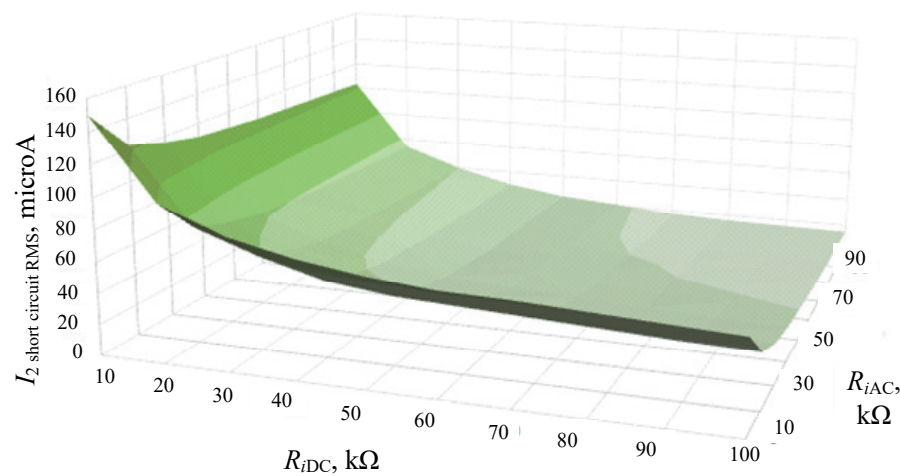

Fig. 6. Dependence of the maximum pole "dead" ground fault current (RMS value) for the rectifier output voltage $U_{12 \text { mean }}=1 \mathrm{~V}$ on the $R_{\mathrm{iDC}}$ and $R_{\mathrm{iAC}}$ ratio.

It is worth to note that similarly to the rectifier output and pole-to-ground voltages, the DC side leakage and ground fault currents contain also an AC component. Its level (i.e., mean value) can be calculated as:

$$
I_{2 \mathrm{AC}}=\sqrt{I_{2 \mathrm{rms}}^{2}-I_{2 \mathrm{mean}}^{2}} .
$$

\section{V.Practical Utilization of RESEARCH RESUlts}

Further, three examples of the utilization of research results are presented.

\section{A. Electric Shock Hazard in the Case of a Human Touching One of Rectifier's Poles}

For the evaluation of electric shock risk it is necessary to determine the current flowing through the human body. When a person with body resistance $R_{\mathrm{H}}$ touches, for example, the negative pole (see Fig. 7), RMS value of the current flowing through his body to the ground can be calculated as a quotient $U_{2 \mathrm{rms}} / R_{\mathrm{H}}$.

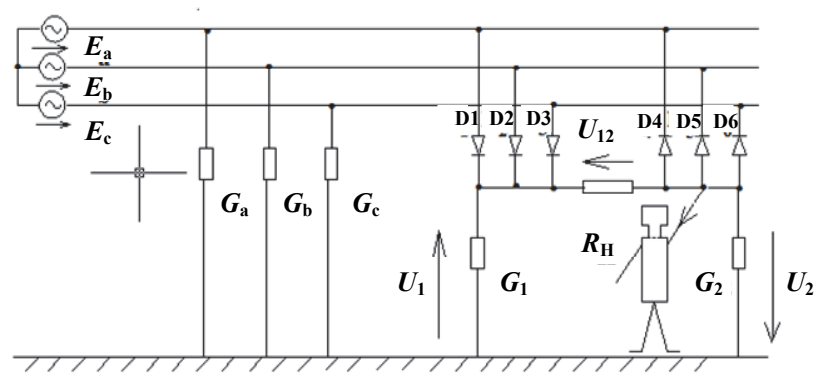

Fig. 7. Electric shock of a human with the body resistance $R_{H}$.

RMS values of the negative pole voltage were obtained in Chapter III. Take note that in the case of electric shock, the resulting insulation conductance of this pole is equal to $G_{2}+1 / R_{\mathrm{H}}$. In Fig. 8, there are plotted curves presenting the dependence of the maximum shock current RMS value on $R_{\text {iDC }}$ for rectifier's output mean voltage $230 \mathrm{~V}$ and two fixed values of $R_{\mathrm{iAC}}$.

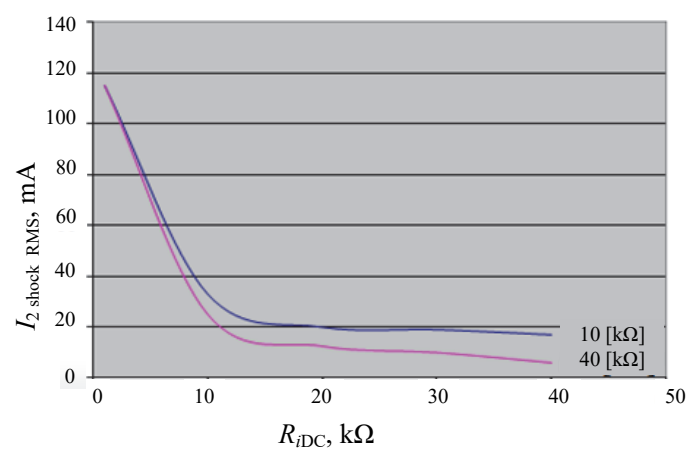

Fig. 8. Dependence of the maximum shock current (RMS value) for the human body resistance $R_{\mathrm{H}}=1 \mathrm{k} \Omega, \mathrm{U}_{12 \text { mean }}=230 \mathrm{~V}$ on $R_{\mathrm{iDC}}$ for two fixed values of $R_{\mathrm{iAC}}=10 \mathrm{k} \Omega ; 40 \mathrm{k} \Omega$.

It should be noted that the permissible level of DC shock current is assumed to be $20 \mathrm{~mA}$.

\section{B. DC Relay Misoperation}

Due to insulation deterioration, an inadvertent lack of the dropout (holding) of a relay in the case of its coil grounding is possible (see Fig. 9). This situation takes place if relay $P$ is energized by closing the $P_{1}$ contact, and then its coil positive terminal gets grounded. When $P_{1}$ contact opens, the relay may not drop out (hold on).

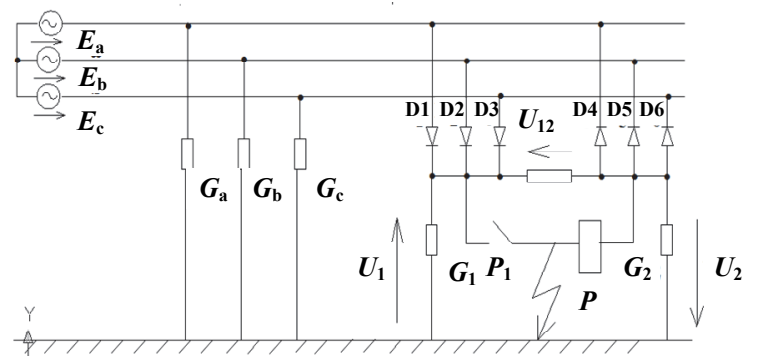

Fig. 9. Inadvertent lack of the dropout (holding) of a relay $P$ in the case of its coil's positive terminal grounding.

Based on relay $P$ holding condition in the worst case $G_{1}=0$ (see (16))

$$
U_{\text {relaymean }}=\frac{G_{\mathrm{iAC}}}{G_{\mathrm{iAC}}+G_{2}+\frac{1}{R_{P}}} \cdot \frac{U_{12 \text { mean }}}{2} \geq U_{P \text { dropout }}
$$

there can be plotted curves presenting the dependence of the maximum mean value of the voltage across relay terminals on $R_{\mathrm{iDC}}=1 / G_{2}$ for the fixed values of $R_{\mathrm{iAC}}$. For testing, a typical Polish magnetoelectric relay $R U-412$ was used (rated data: voltage $220 \mathrm{VDC}$, dropout voltage $U_{P \text { dropout }}=150 \mathrm{~V}$, coil resistance $R_{P}=16.4 \mathrm{k} \Omega$ ). The drawing shows that the risk of this relay's inadvertent holding appears with $R_{\mathrm{iDC}}$ not more than $8 \mathrm{k} \Omega$. 


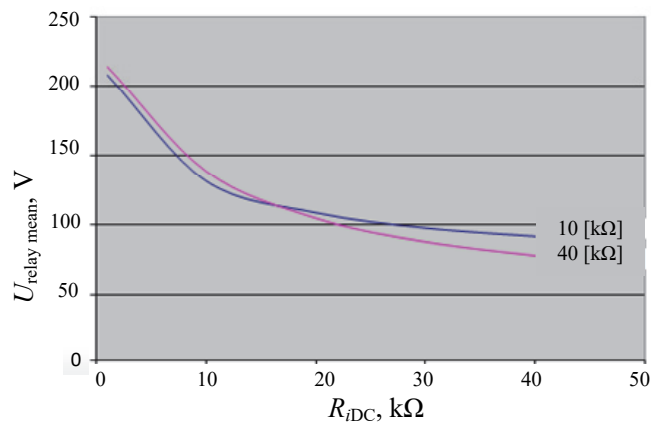

Fig. 10. Dependence of the maximum mean value of voltage across $R U-412$ relay terminals on $R_{\mathrm{iDC}}$ for $\mathrm{U}_{12 \text { mean }}=230 \mathrm{~V}$ and two fixed values of $R_{\mathrm{iAC}}$

From Figs 8 and 10 it can be concluded that insulation resistance at DC side of the rectifier is decisive for both hazards. Insulation condition at $\mathrm{AC}$ side plays a minor role.

\section{Insulation Resistance Measurement}

The mean values of voltages in rectifier's output circuit can be used to determine the whole AC/DC IT network's insulation equivalent resistance $R_{\mathrm{i}}$ defined as

$$
R_{\mathrm{i}}=\frac{1}{G_{\mathrm{iAC}}+G_{\mathrm{iDC}}}=\frac{1}{G_{\mathrm{a}}+G_{\mathrm{b}}+G_{\mathrm{c}}+G_{1}+G_{2}} .
$$

This measuring procedure is a well-known method of three readouts of one DC voltmeter. These are three successive measurements of the mean values of voltage: 1) $U_{12 \text { mean }}$, 2) $U_{1 \text { mean }}$ with a test resistor $R_{0}$ connected between pole 1 and ground, 3) $U_{2 \text { mean }}$ with a test resistor $R_{0}$ connected between pole 2 and ground. According to formulas (15) and (16), $U_{1 \text { mean }}$ and $U_{2 \text { mean }}$ are equal to

$$
\begin{aligned}
& U_{1 \text { mean }}=\frac{G_{\mathrm{a}}+G_{\mathrm{b}}+G_{\mathrm{c}}+2 G_{2}}{G_{\mathrm{a}}+G_{\mathrm{b}}+G_{\mathrm{c}}+G_{1}+G_{2}+G_{0}} \cdot \frac{U_{12 \text { mean }}}{2}, \\
& U_{2 \text { mean }}=\frac{G_{\mathrm{a}}+G_{\mathrm{b}}+G_{\mathrm{c}}+2 G_{1}}{G_{\mathrm{a}}+G_{\mathrm{b}}+G_{\mathrm{c}}+G_{1}+G_{2}+G_{0}} \cdot \frac{U_{12 \text { mean }}}{2} .
\end{aligned}
$$

Transforming these equations, the final formula is obtained:

$$
R_{\mathrm{i}}=R_{0} \frac{U_{12 \text { mean }}-U_{1 \text { mean }}-U_{2 \text { mean }}}{U_{1 \text { mean }}+U_{2 \text { mean }}} .
$$

It should be noted that (23) is valid also for any asymmetrical three-phase supply of diode rectifiers. It can be used for the determination of insulation equivalent resistance in DC circuits too.

\section{CONCLUSION}

1. The mean value of diode rectifier's output voltage fed with three-phase asymmetrical voltages is determined. It is proportional to the sum of all line-to-line voltages.

2 . The maximum level of any pole-to-ground voltage mean value is obtained when the entire DC side insulation leakage conductance is lumped at the opposite pole. The distribution of the AC side insulation leakage conductances between single phases is of no importance.

3. Maximum leakage and ground fault currents at DC side can be determined with the help of enclosed tables (with the use of linear interpolation for insulation resistances not given there) and diagrams.

4. The measurement of voltages in the output circuits of rectifiers can be utilized for analytical determination of the insulation resistance of the whole AC/DC IT network.

\section{REFERENCES}

[1]P. Olszowiec, "Insulation Measurement and Supervision in Live AC and DC Unearthed Systems," Lecture Notes in Electrical Engineering, 2013. https://doi.org/10.1007/978-3-642-29755-7

[2] W. Hofheinz, Protective Measures with Insulation Monitoring, VDEVerlag, 1998.

[3]P. Olszowiec, "Evaluation of Variation Ranges of Phase Voltages and Ground Currents in Three-Phase Unearthed Networks With Diode Rectifiers," Power and Electrical Engineering, vol. 33, pp. 51-55, 2016. https://doi.org/10.7250/pee.2016.010

[4]P. Olszowiec, "Unconventional Methods of Analyzing Diode Rectifiers with Asymmetrical Supply," Computational Problems of Electrical Engineering, vol. 4, no. 2, pp. 33-36, 2014

[5]P. Olszowiec, „O wyznaczaniu napięć trójfazowych prostowników diodowych," Wiadomości Elektrotechniczne, vol. 10, pp. 20-21, 2015. 